\title{
Profesionales del derecho, activismo jurídico y creación de nuevos derechos. Hacia una mirada comprensiva del derecho desde las ciencias sociales
}

\author{
VIRGINIA VECCHIOLI
}

$\mathrm{E}$ ste número de la Revista Política reúne artículos que aportan a la comprensión de las complejas relaciones entre el derecho y la política. Los ocho trabajos que integran esta publicación ofrecen al lector un corpus de datos empíricos sustantivos y una mirada provocadora sobre las formas de constitución recíproca entre ambas esferas poniendo en el centro del análisis los procesos de formación de nuevas elites jurídicas (Engelmann), de innovación en los sistemas judiciales (Palacios, Herrero), de institución de nuevos derechos, tradiciones jurídicas y sujetos de derecho (Guilhot, Gerardi y Zibecchi) y de movilización del derecho y de sus profesionales en la conformación de diversas causas públicas (Israël, Ríos Petrarca, Alves Maciel y Da Silva Brito Prata).

En la integración de este dossier se ha privilegiado la diversidad de casos empíricos presentados (desde abogados de corporaciones empresarias, defensores de derechos humanos y miembros del poder judicial hasta expertos transnacionales en programas de reformas del derecho), la diversidad de tradiciones disciplinarias (juristas, sociólogos, cientistas políticos, antropólogos) y la diversidad de contextos espaciales y temporales (desde la Segunda Guerra Mundial y el periodo de posguerra hasta las recientes reformas legislativas de comienzos del siglo XXI en contextos tan diversos como Rio Grande do Sul, Estados Unidos, París y Buenos Aires). El propósito de este ensamblado heterogéneo de trabajos no es el de proponer una apología de la diversidad de agentes y de actividades vinculadas al derecho sino el de mostrar la productividad de una perspectiva de análisis.

Esto implica, en primer lugar, que no encontrará el lector artículos centrados en la discusión dogmática o abstracta del derecho, lo que supuso dejar de lado propuestas centradas en los discursos jurídicos o en las instituciones per se, desvinculadas de sus agentes y prácticas constitutivas específicas. En segundo lugar, se privilegió la selección de trabajos que suponen una reflexión distanciada de la manera en que los propios interesados reflexionan sobre estas temáticas afirmando el valor de aquel principio básico de las ciencias sociales que señala la necesidad de trascender las explicaciones fundadas en la voluntad de los propios agentes. Como señala Remi Lenoir, para sortear la falacia de concretud contenida en sus propias definiciones, el investigador debe trascender el poder que emana de las construcciones intelectuales elaboradas por los propios juristas sobre su actividad y sobre las razones tan razonables que explican sus acciones y "obstinarse en describir al derecho 'tal como éste es practicado'” (Lenoir, 2002: 205). A través de la edición de este dossier se pretende acercar al lector un conjunto 
de artículos cuyo valor principal reside en llevar adelante esta 'obstinación' de comprender al derecho como un hecho social. Se busca, con ello, promover una mirada sociológicamente comprensiva sobre esta práctica social en la producción de las ciencias sociales de América Latina, desplazando el eje de atención de lo que el derecho debería ser a lo que el derecho efectivamente es.

Quitar a los juristas el monopolio de los principios de comprensión de sus propias prácticas supone un doble movimiento: por un lado, tomar al lenguaje jurídico y a sus productores como si fueran exóticos y extraños a condición de despojarlo de la impronta de 'obviedad' y 'necesidad' que tiene su intervención para el sentido común y para sus expertos (Bourdieu, 1998) para preguntarnos sobre las condiciones que hacen posible su intervención ante situaciones de conflicto social y político -este distanciamiento del cotidiano no resulta demasiado difícil si consideramos que el derecho no es fácilmente comprensible para el gran público como tampoco lo son las reglas matrimoniales bantúes o las ceremonias de iniciación en Tierra del Fuego (Lenoir, 2002:262). Pero, por otro lado, exige tomar las prácticas e interpretaciones de sus especialistas como la materia prima a partir de la cual construir y explicitar el funcionamiento de este universo, haciéndolo inteligible mediante la comprensión de sus afinidades con otras esferas de acción. Este procedimiento permite eludir la trampa que acecha al investigador en este terreno, la exotización del derecho, para mostrar, en definitiva, cómo aquello que sucede dentro de este espacio profesional también sucede en el contexto social más amplio en el cual se desarrolla. Los problemas derivados de la esencialización del derecho como práctica social pueden ser evitados cuando se piensa este campo profesional a partir de los mismos principios de análisis y herramientas metodológicas que las disciplinas sociales han construido para dar cuenta de otros espacios y relaciones.

En este sentido, la principal preocupación en el armado de este dossier ha sido promover la producción de trabajos que tomen la categoría derecho como una categoría etnográfica -si se me permite este 'antropocentrismo'-, como una categoría que no preexiste a las formas en que los nativos, los propios especialistas, construyen el derecho en contextos social e históricamente situados. Esta indicación importa en tanto la reflexión sobre el derecho y los sistemas de justicia, especialmente en América Latina, se encuentra cuasi monopolizada por los propios juristas y magistrados, quienes, al intervenir en las disputas al interior de su propio campo, lo hacen a través de la producción de textos académicos y eruditos. Esto es válido, inclusive, para aquellos juristas llamados 'alternativos' que se apoyan en las ciencias sociales para asumir una posición 'crítica' respecto del derecho, dado que, ellos también, son parte interesada en definir los criterios de funcionamiento de este campo. Es preciso advertir que, mediante una profusa producción intelectual, estos expertos aspiran a delimitar tanto los temas y los términos del debate disciplinario como los criterios legítimos para intervenir en el mismo. En el marco de estos debates, la vinculación entre derecho y política aparece frecuentemente estigmatizada y condenada o, en un contrapunto perfecto, considerada desde una perspectiva exclusivamente laudatoria. En definitiva, 
ambas definiciones expresan más el interés de quien las enuncia por sentar posición dentro del propio campo que una preocupación por comprender su lógica de funcionamiento, donde la vinculación con la política no sea objeto de evaluación moral sino condición de comprensión del derecho como práctica social.

Cuando este juego de estrategias y posicionamientos, que es constitutivo del propio campo del derecho, se desliza inadvertidamente en el análisis de los cientistas sociales, el resultado es una visión normativa del derecho en la cual las distintas piezas y movimientos en el tablero acaban delineando las temáticas y los problemas legítimos de investigación, replicando en el campo académico las jugadas que realizan los especialistas del derecho dentro de su propio mundo social ${ }^{1}$. Esto explica que las complejas relaciones entre derecho y politica aparezcan en estos análisis frecuentemente como sobreentendidas o limitadas en su comprensión por las mismas perspectivas laudatorias o condenatorias que son parte de las demandas formuladas por los propios especialistas sobre la necesidad de contar, por ejemplo, con un poder judicial 'independiente' gracias a la puesta en marcha de procesos de 'reforma' judicial. Como advierte el propio Daniel Palacios respecto de la literatura sobre reforma judicial en América Latina, ésta ha sido producida, por lo general, por los mismos actores que la promueven y por quienes desean abrir una discusión en torno al rumbo que deberían tomar tales procesos: "Así estos análisis se circunscriben (...) a la dirección que debiera tomar la política de reforma judicial en la región, contribuyendo de esta forma a la reproducción de este campo de reformas" (Palacios, página 44).

La participación de los cientistas sociales en los propios procesos políticos y jurídicos que dan lugar a la investigación y a la reflexión erudita hace que el propósito de comprender al derecho como un hecho social no sea, en absoluto, una operación sencilla dado que, frecuentemente, el investigador comparte la perspectiva de sus nativos sobre cómo debería funcionar la justicia y cuál debería ser la contribución del derecho al funcionamiento del sistema político. Este desafío se verifica en relación con varios de los trabajos incluidos en este dossier, tanto aquellos referidos a la introducción de reformas judiciales y al surgimiento de nuevas 'culturas jurídicas' en América Latina como a los que problematizan la categoría 'derechos humanos' o 'resistencia'. Los primeros porque destacan la estrecha vinculación entre el contexto de políticas neoliberales, la intervención de instituciones transnacionales como el Banco Mundial y la difusión del Estado de Derecho como ideario político para los países de la región. ¿Qué complejos procesos sociales y políticos se desarrollan al interior de los distintos Estados nacionales a partir de la adopción de este ideario de la rule of law que no estaban

\footnotetext{
Ésta es un poco la situación en la que se encuentran la sociología y la antropología jurídicas. Entre la numerosa literatura que señala las limitaciones de la 'sociología jurídica' se encuentra el trabajo de Bancaud y Dezalay (1984). El valor del mismo consiste en que proporciona no sólo una crítica de este subcampo disciplinario sino que brinda, a su vez, una visión comprensiva del mismo, explicitando la lógica y los principios que instituyen las posiciones adoptadas por los juristas sociólogos y los sociólogos del derecho.
} 
previstos inicialmente en los objetivos de estas reformas? ¿Cómo contribuyen estos procesos a la creación de nuevas jerarquías y desigualdades? La segunda línea de trabajos también supone un desafío significativo porque abordan temas particularmente sensibles para el contexto europeo y de América Latina como es la Resistencia contra el régimen nazi y la lucha por los derechos humanos en contextos dictatoriales revelando la complejidad interna de estos espacios y las transformaciones operadas en el tiempo. Al suspender las explicaciones nativas sobre estos hechos que remiten, básicamente, al coraje individual o al éxito colectivo de las acciones emprendidas por organizaciones no gubernamentales, los artículos destacan las mediaciones existentes entre los hechos objetivos de violencia y desprotección a la vida y el reconocimiento de estos derechos en el ámbito estatal e interestatal. ¿Qué procesos sociales posibilitan el reconocimiento jurídico de los derechos humanos y su institución en políticas de Estado? ¿Qué sentidos múltiples se ponen en juego cuando se apela a la categoría derechos humanos? ¿Es ésta una categoría unívoca, universal, atemporal y, por lo tanto, 'apolítica'? ¿Qué implicancias tiene para las aspiraciones de reconocimiento universal por parte de sus activistas identificar su carácter situado y el hecho de estar fundado en proyectos políticos específicos?

Los artículos reunidos en este dossier proponen habilitar este tipo de interrogantes y desafíos eludiendo con maestría las posiciones laudatorias o condenatorias respecto de los vínculos entre derecho y política para mostrar productivamente las múltiples y complejas relaciones entre estas esferas. Esto es posible gracias a que los autores incorporan dimensiones de análisis que exceden al propio derecho bajo el convencimiento de que éste no puede contener en sí mismo sus propios principios de explicación.

\section{Los artículos del dossier: desafíos teóricos y metodológicos}

En la integración de este dossier se pone en evidencia cómo nuevas líneas de trabajo comienzan a abrirse paso a partir de la puesta en foco de las complejas relaciones entre derecho y política. Los artículos que abordan la temática del activismo jurídico son un primer ejemplo de este punto. Lo mismo ocurre con los trabajos que ponen de relieve las condiciones de surgimiento de nuevas 'culturas jurídicas' o aquellos que muestran el impacto de las sentencias del poder judicial en la formulación e implementación de políticas públicas.

Los artículos referidos al activismo jurídico ilustran cómo el propio ejercicio profesional del derecho puede constituirse en una forma significativa de intervención política al mostrar el papel que cumplen sus agentes en la traducción de causas militantes en causas profesionales. Como reseña con propiedad Fernanda Ríos Petrarca, esta singular articulación entre derecho y activismo intenta, por un lado, sacar provecho del vasto conjunto de trabajos orientados al estudio de los procesos de compromiso y movilización colectiva y, por otro, de los estudios sobre la formación de grupos profesionales y su dinámica interna de organización y 
funcionamiento, que se insertan en las discusiones de la llamada "sociología de las profesiones". Y ello porque al poner el foco en este tipo de activismo se evidencia que quienes lo asumen son profesionales que se constituyen como tales a partir de su compromiso político y del ejercicio profesional del derecho. Las particularidades que en cada caso adquiere esta configuración dan relevancia a principios distintivos de reclutamiento, de adhesión y de representación que suponen, a su vez, formas específicas de hacer y pensar el derecho y la política.

En los casos tratados en este dossier es posible identificar la participación de activistas y especialistas en derecho en la promoción de causas públicas, en la invención de repertorios de jurisprudencia, en la formulación e implementación de políticas de Estado y en el impulso a programas de reforma del sistema de justicia, procesos todos que conllevan formas particulares de vinculación de los expertos en derecho con el poder ejecutivo y legislativo y cuya lógica de articulación es necesario develar como condición de comprensión de la propia práctica jurídica. El activismo jurídico supone formas específicas de reclutamiento y de movilización de magistrados, profesores de derecho, juristas, expertos, diplomáticos y abogados, la apertura de nuevos canales de ingreso a la profesión y al espacio del poder de Estado y la conformación de nuevas elites jurídicas o la transformación de las existentes sobre nuevos principios de legitimación. Es por ello que la reconversión del derecho en un recurso para la movilización en defensa de causas públicas tiene impacto no sólo en el espacio público, sino también dentro del propio campo profesional en tanto da lugar a diversas formas de diversificación del espacio jurídico, a la creación de instancias específicas de formación académica y profesional para el entrenamiento de los jóvenes profesionales en la representación de estas causas, como lo demuestra al auge de las "clínicas jurídicas" y posgrados y especializaciones en derecho, y el surgimiento de nuevos requerimientos técnicos y profesionales relacionados con la actuación de los profesionales del derecho dentro de las agencias del Estado, los movimientos sociales o las agencias de cooperación internacional.

Para comprender la articulación entre un proyecto militante y un tipo específico de competencia experta es necesario atender al proceso que articula dimensiones de distinto orden, como la vocación, la política y la profesión y esferas también diversas como las organizaciones civiles, la universidad, el Estado y la esfera transnacional e interestatal. La complejidad de estas dimensiones justifica que se consideren en detalle los cambios que tienen lugar en el espacio del derecho y la política destacándose entonces la productividad de los enfoques orientados a poner en evidencia los programas curriculares de las facultades de derecho, los espacios de producción doctrinaria (la irrupción de nuevas revistas, institutos de estudios, cátedras, etc.), el perfil de los estudios de abogados, sus principios de reclutamiento, los circuitos locales e internacionales de circulación de estos expertos, las trayectorias políticas y profesionales de sus especialistas, sus estrategias de legitimación, sus espacios de socialización, los modelos e idearios políticos que sustentan sus prácticas y la manera en que se vinculan tanto en el espacio local como transnacional. 
Estas formas de activismo también impactan en el propio mundo asociativo de manera que se impone tener en cuenta en el análisis las estrategias de apropiación del espacio jurídico por parte de los movimientos sociales, dimensión destacada especialmente en los trabajos de Carla Zibecchi y Natalia Gherardi y de Débora Alves Maciel y Ana Paula da Silva Brito Prata. Y esto porque la articulación de los movimientos sociales con el mundo del derecho produce efectos concretos en la manera en que se procesan e interpretan los conflictos políticos, en la definición de los agentes autorizados para dar cuenta de ellos, en la conformación del repertorio de acción de los movimientos y en las estrategias de movilización que adoptan, en la profesionalización del activismo y en la conversión de éste en un asunto de competencia experta. El indagar en estas dimensiones y ponerlas en relación con la estructura de oportunidades políticas es lo que permite desnaturalizar la apelación al derecho como instancia de resolución de conflictos políticos y abrir la puerta al examen detallado del proceso social que lo hace posible, como ilustran los casos referidos a la resistencia francesa durante la ocupación alemana (Israël) y la creación de nuevos repertorios jurídicos relativos a la violencia de género (Alves Maciel y Da Silva Brito Prata). En el examen detallado de este proceso, la adopción de una perspectiva procesual se vuelve un elemento clave a la hora de comprender los ciclos de adhesión a la causa, las heterogeneidades del compromiso, las condiciones de profesionalización del mismo y la lógica a partir de la cual el derecho se convierte en un recurso de acción militante.

La complejidad de estos procesos se pone claramente de manifiesto en los análisis orientados a comprender las reformas de la justicia ya que estos programas suponen, en efecto, algo más que un simple cambio de reglas. Involucran procesos sociales que activan y articulan un complejo entramado de actores: especialistas en derecho pero también cuadros dirigentes de asociaciones civiles y ONGs, asociaciones profesionales, redes de expertos, miembros del poder judicial, funcionarios públicos, dirigentes partidarios y agencias de cooperación internacional (como el Banco Mundial o el Banco Interamericano de Desarrollo, BID), intereses, modelos, valores y fuentes de financiamiento muy diversas. Esta heterogeneidad de intereses, posiciones y marcos interpretativos da lugar a visiones muchas veces conflictivas en cuanto a los objetivos de las reformas y a los medios para instrumentarlas.

El trabajo de Fabiano Engelmann sobre las esferas de socialización de los abogados de negocios en Brasil se enmarca en las reflexiones más generales para el contexto latinoamericano referidas al impacto de las políticas neoliberales implementadas en la región durante los años 90 (privatizaciones de los servicios públicos, internacionalización de los mercados financieros, endeudamiento público, reformas constitucionales, etc.). A lo largo de estos años el espacio regional fue profundamente re-estructurado por políticas que se desarrollaron en sintonía con el llamado 'consenso de Washington'. En este contexto emergieron demandas de reforma de la justicia ya que -desde la perspectiva de los Bancos Multilaterales de Desarrollo (BMDs-Banco Mundial, BID)- las reformas macroeconómicas sólo producen resultados favorables y duraderos si son acompañadas por reformas en 
el sistema político y jurídico. En este marco, el Poder Judicial, su legitimidad y eficiencia, comenzó a ser reconocido como una pieza clave para el buen gobierno en general y para un eficiente funcionamiento de los mercados en particular. Como señala el propio Engelmann, los modelos de reforma de las instituciones judiciales que contemplan la unión entre la "democracia", la racionalidad de las instituciones y su afinidad con el orden económico pueden ser sintetizados en las diversas proposiciones del ideario de la rule of law presente en las evaluaciones y en los documentos oficiales de organismos internacionales, entre los cuales se destaca el Banco Mundial. Este ideario impactó profundamente al interior del espacio jurídico ya que posibilitó abrir la agenda de las reformas legales y permitió el ingreso de los sectores más cosmopolitas de la profesión que tiene sus contactos y experticias internacionales valorizadas. En un mismo sentido, impulsó a los gobiernos locales a adecuar los modelos jurídicos con el propósito de ofrecer un ambiente favorable a la atracción de inversiones extranjeras.

$\mathrm{Si}$ estos procesos más generales relativos a las politicas neoliberales han sido desarrollados abundantemente por la literatura especializada, lo que el trabajo de Engelmann nos revela como elemento innovador es el impacto de estos procesos al interior del mundo del derecho: internacionalización de la profesión, surgimiento de nuevos valores y principios de legitimación, aparición de nuevas especializaciones profesionales, cambios en el currículo universitario y cambios en las relaciones de poder entre los distintos grupos que integran este espacio profesional. Analizando la producción doctrinaria sobre derecho de negocios, el perfil de las principales sociedades de abogados y sus socios y el espacio de las cámaras de mediación y arbitraje, Engelmann muestra con contundencia cómo la creciente legitimación de una cultura jurídica de "mercado" junto con el armado de mecanismos legales favorables al derecho empresarial internacional se produce en estrecha afinidad con los modelos exportados al espacio regional por instituciones transnacionales como el Banco Mundial, donde lo que está en juego es la propia definición del Estado como regulador de las prácticas económicas así como de los modelos de gestión del sistema judicial. En este contexto, esta cultura jurídica puede ser comprendida como parte de una batalla simbólica que permea el espacio más amplio de producción de doctrinas jurídicas y que opone a los intérpretes de la Constitución, posicionados en las carreras del Estado, a los juristas y economistas que invierten en la producción de una "cosmovisión jurídica de mercado" apoyada en el crecimiento de las grandes sociedades de abogados y en las prácticas de mediación y arbitraje.

En estrecha relación con este artículo, el trabajo de Daniel Palacios sobre los procesos de reforma judicial en Chile pone en evidencia que estas reformas suponen algo más que un simple cambio de reglas: involucran procesos sociales que activan y articulan un complejo entramado de actores, especialistas en derecho pero también cuadros dirigentes de asociaciones civiles y ONGs, asociaciones profesionales, redes de expertos, miembros del poder judicial, funcionarios públicos, dirigentes partidarios y agencias de cooperación internacional (como el Banco Mundial o el BID), intereses, modelos, valores y fuentes de financiamiento muy 
diversas. Esta heterogeneidad de intereses, posiciones y marcos interpretativos da lugar a visiones muchas veces conflictivas en cuanto a los objetivos de las reformas y los medios para instrumentarlas, y justifica que el análisis destaque las estrechas relaciones entre el espacio del derecho y la política como condición de comprensión de las lógicas con las que funciona este universo social.

La articulación de estas dimensiones es la clave del análisis de Palacios, quien inicia su trabajo con una reconstrucción minuciosa de las trayectorias iniciales de los agentes que promueven la reforma procesal penal partiendo de la posición que ocupaban en la estructura del campo jurídico chileno en el año 2000, de sus relaciones mutuas así como del contexto político marcado por el fin de la dictadura y la transición democrática. Palacios muestra cómo estas trayectorias les permiten a los promotores de la reforma insertarse en espacios institucionales desde los que podrán movilizar una serie de recursos políticos y académicos que posicionarán a esta reforma dentro de la agenda de políticas del Estado chileno. Uno de los principales efectos de estos procesos sobre el campo del derecho será la conformación de un saber de Estado resultado de la interacción del espacio internacional con el local, que se instituye en un capital decisivo a la hora de posicionar a estos agentes en el espacio jurídico y político chileno. De la misma manera que se advirtió para el caso brasileño, en este proceso tendrán una importancia crítica la importación de ideas del exterior y de enfoques de modernización política pública y judicial, viabilizada por la circulación de agentes y expertos en el espacio transnacional.

Una entrada diferente al espacio de producción de políticas de Estado la constituye el artículo de Álvaro Herrero. A través del análisis de una serie de sentencias de la Corte Suprema de Justicia de la Nación de Argentina dictadas a partir de 2005 y relativas a casos 'previsionales', el autor se propone mostrar la notoria influencia del accionar de la Corte en el diseño de la política pública previsional argentina, las tensiones que están envueltas en este proceso y sus resultados. En un trabajo meticulosamente documentado, Herrero destaca el examen de algunos casos ejemplares para mostrar en toda su complejidad la encrucijada entre el poder político y judicial, poniendo en evidencia cómo las sentencias de la Corte Suprema de Justicia de la Nación contribuyeron a generar cambios concretos en la forma en que los poderes Ejecutivo y Legislativo -a través de las agencias correspondientes- despliegan y articulan sus acciones en el área previsional. Las acciones iniciadas por la Corte culminaron con la aprobación de un nuevo marco regulatorio aprobado por el Congreso, marcando un cambio respecto a los patrones históricos de relación entre el Poder Judicial y el Poder Ejecutivo.

La decisiva intervención del poder judicial en la consagración y reconocimiento de derechos es analizada también en detalle por Natalia Gherardi y Carla Zibecchi. Apelando a la categoría "activismo judicial" las autoras muestran cómo se articulan derecho y política, específicamente en relación con casos de violación de derechos sociales tratados ante los tribunales de la Ciudad de Buenos Aires. En su trabajo las autoras se proponen revisar qué condiciones posibilitan la politización del derecho y su movilización como un repertorio de acción pública. En la 
comprensión de este proceso resultan claves la articulación entre una asociación civil integrada por profesionales del derecho (la Asociación Civil por la Igualdad y la Justicia), la actuación de los jueces capitalinos, la incorporación de normas del derecho internacional al derecho positivo local y las acciones del gobierno de la ciudad. Al igual que Herrero, muestran la decisiva intervención del poder judicial en la etapa de cumplimiento de las sentencias, en este caso relativas a la aplicación de programas de cuidado familiar, poniendo de relevancia la agencia de este actor en la implementación de políticas públicas.

Continuando con el análisis del activismo jurídico en materias relativas al género, el trabajo de Débora Alves Maciel y Ana Paula da Silva Brito Prata nos presenta una reconstrucción de la movilización pública que resultó en la sanción de una ley de protección contra la violencia de género. La investigación empírica que las autoras desarrollan contempla el análisis de las múltiples dimensiones de la acción colectiva -la estructura de oportunidades políticas, las carreras militantes, los frames y las estrategias de movilización- para explicar la emergencia, la dinámica y los resultados jurídico-institucionales de la campaña. En la reconstrucción de este activismo de género se destaca la importancia de considerar en el análisis las formas de articulación entre organizaciones de la sociedad civil, las instituciones interestatales (como la Comisión Interamericana de Derechos Humanos) y las asociaciones civiles que nuclean a activistas del derecho (como es para este caso el Cejil). Comprender las condiciones de posibilidad de esta nueva ley supone ir más allá del reconocimiento objetivo de la violencia contra la mujer. Esto es algo que funda la perspectiva de las autoras de este artículo al mostrar todo el trabajo que media entre los episodios individuales y privados de violencia contra la mujer y el reconocimiento público de sus derechos. Junto al uso de recursos jurídicos, las autoras exploran la dimensión simbólica de los usos políticos del derecho. Como señalan en su trabajo, a través de este análisis "Buscamos mostrar cómo este episodio de movilización legal propicia la comprensión tanto de las múltiples dimensiones del activismo jurídico (político-institucional/ estratégica y cultural/simbólica) como de sus efectos potenciales sobre la expansión del derecho en la regulación de las relaciones sociales en las sociedades contemporáneas".

El artículo de Liora Israël presenta tres modalidades de resistencia al poder fundadas en el derecho en el contexto de la Resistencia Francesa a la ocupación alemana durante la Segunda Guerra Mundial. La autora muestra el compromiso de abogados y magistrados en su lucha contra el poder de ocupación reconociendo, al mismo tiempo, la diversidad de posiciones adoptadas por estos profesionales del derecho en este contexto político de extrema violencia, como una manera de hacer más complejo el análisis de las relaciones entre derecho y política. Si frente al mismo hecho histórico de la ocupación alemana los profesionales del derecho no adoptaron las mismas estrategias de resistencia, ¿cómo explicar estas diferencias? Al intentar una respuesta a este interrogante que supere la explicación fundada en supuestas diferencias personales (las explicaciones nativas fundadas en el coraje, el sacrificio, etc.), la autora pone en marcha un extraordinario 
esfuerzo de desnaturalización de este activismo y de restitución de esta práctica engendrada en un contexto de extrema violencia a la lógica de funcionamiento del mundo social. Como resultado de este trabajo, podemos reconocer la pluralidad de usos del derecho y de posiciones dentro de este universo.

Este conjunto de artículos introducen, de alguna manera, los trabajos de Fernanda Ríos Petrarca y de Nicolas Guilhot referidos al activismo en derechos humanos. Ambos trabajos muestran, en primer lugar, el sentido necesariamente situado de la categoría genérica 'derechos humanos'. En el contexto brasileño, la defensa de los derechos humanos aparece asociada a la defensa de causas raciales. En el contexto de los EE.UU. y durante el periodo que va desde el fin de la administración Carter a la llegada al poder de Reagan, Guilhot nos presenta las diferencias de sentido contenidas en la misma categoría nativa, iluminando la necesaria condición histórica de unos derechos humanos que se pretende universales y atemporales. El análisis de Ríos Petrarca se basa en el examen de la relación entre la defensa de causas colectivas vinculadas a la cuestión racial y la actuación profesional basada en una experticia jurídica en el sur de Brasil, reconstruyendo las trayectorias de un conjunto de abogados que actúan profesionalmente en defensa de la igualdad racial en el estado de Rio Grande do Sul. Lo que este estudio permite mostrar es que los principios de identificación étnico-racial y el compromiso militante son fundamentales para conformar sus concepciones y prácticas profesionales. El artículo de Nicolas Guilhot presenta una de las aristas más complejas sobre el activismo en derechos humanos, resultado de la perspectiva metodológicamente distanciada adoptada por el autor. Esta operación de desnaturalización desafía el sentido común aceptado tanto por activistas como por académicos sobre los principios de constitución de este universo social y genera desplazamientos respecto de los marcos y límites de lo decible en términos de activismo en derechos humanos, fundamentalmente el carácter político del mismo.

\section{Consideraciones finales: para pensar más allá del dossier}

La lectura cruzada de los distintos artículos que integran el dossier permite avanzar en nuevos interrogantes y caminos de análisis. Resulta especialmente interesante considerar las formas novedosas en que el sistema de justicia incide en la formulación e implementación de políticas públicas, como en el caso desarrollado por Herrero sobre la política previsional en la Argentina de los años 90 o el presentado por Zibecchi y Gherardi respecto del derecho al cuidado. Los artículos de Álvaro Herrero y de Liora Israël apoyados empíricamente sobre casos tan diversos como el funcionamiento de la Corte Suprema Argentina en un contexto democrático y la resistencia de los magistrados franceses al gobierno de Vichy, dejan entrever con claridad la importancia de trascender las visiones maniqueas sobre el funcionamiento de la magistratura a favor de una perspectiva que incluya, productivamente, un análisis de la heterogeneidad de posiciones, trayectorias y contextos en que los magistrados desarrollan su trabajo. 
Si los artículos mencionados invierten en mostrar con mucha eficacia la importancia de las decisiones del poder judicial a la hora de hacer efectiva la legislación, también es legítimo preguntarse, inversamente, sobre el efecto o impacto de las distintas modalidades de intervención del Estado en a) la creación de condiciones que hacen posibles la movilización y el activismo jurídico, como se pone en evidencia en el trabajo de Ríos Petrarca relativo a la creación del Programa SOS Racismo en Brasil, y b) la manera en que esta política de Estado contribuye a institucionalizar y profesionalizar el espacio de activismo vinculado a la defensa de derechos colectivos, creando nuevos puestos de trabajo tanto dentro como fuera del ámbito oficial. Estos procesos impactan en la creación de nuevos estudios jurídicos especializados en la defensa de ciertas causas o en el litigio de temas específicos, lo que sugiere la necesidad de explorar también esta dimensión del ejercicio de la profesión. Lo mismo vale para la comprensión del impacto de la esfera transnacional en las estrategias locales asumidas por los magistrados, una dimensión que valdría la pena explorar en futuros trabajos. Al señalar la importancia de poner la mirada en el entrecruzamiento de espacios y en la porosidad de las fronteras entre el ámbito estatal y las asociaciones civiles, vale la pena mencionar los riesgos de instalar una fisura entre estos espacios y entre activismo jurídico y asociativo cuando se verifica en los análisis aquí presentados que los propios integrantes de las ONGs que impulsan el reconocimiento de derechos son ellos mismos abogados que participan, a su vez, en diversos programas y políticas de Estado.

Igualmente provocador resulta asociar los perfiles antitéticos de los abogados de negocios y los abogados vinculados a organizaciones y movimientos sociales para preguntarse sobre las semejanzas y diferencias entre sus lógicas de acción y los puntos en los cuales sus diferentes lógicas se intersectan. Trabajos previos de Bryant Garth, Yves Dezalay (2008, 2002), Mikael Madsen (2004) y del propio Nicolas Guilhot (2004) sugieren que entre las condiciones para intervenir en el espacio del activismo en causas humanitarias está el adoptar una lógica de empresa a fin de poder lidiar exitosamente en el cada vez más competitivo mercado filantrópico transnacional. Por otro lado, es posible reconocer para el caso de América Latina que, a la manera de los buffetes norteamericanos, los grandes estudios de abogados de negocios comienzan progresivamente a realizar trabajo pro-bono en defensa de causas de interés público, lo que sugiere el impacto de la importación de modelos profesionales y el surgimiento en el contexto local de nuevos principios de legitimación.

Todos estos procesos redundan en cambios en cuanto a las condiciones de ingreso de nuevos miembros al campo del derecho. Al poner el énfasis en las trayectorias de los abogados comprometidos en la defensa de las causas raciales o en la implementación de programas de reforma de la justicia, los trabajos de Ríos Petrarca y Palacios muestran con eficacia el ingreso a este espacio profesional de sujetos que no pertenecen a las elites jurídicas tradicionales, lo que sugiere también la importancia, otra vez, de trascender las visiones maniqueas sobre este universo como integrado exclusivamente por sectores tradicionales. El 
trabajo de Palacios muestra específicamente que sujetos con otras propiedades sociales hacen su ingreso a la profesión ocupando espacios centrales a la configuración de poder -y no necesariamente marginales como podría deducirse en forma simplificada de la consideración de su origen social. Inversamente, en el campo del derecho de negocios, sectores tradicionales compiten en función de distintos principios de legitimación -los diplomas obtenidos en universidades tradicionales vs. en el exterior, mostrando que al interior de este segmento profesional aquellos con un capital social más cosmopolita tendrán más éxito en definir las condiciones de funcionamiento del derecho de negocios. Estos requisitos -un alto capital cultural y de relaciones- son también los que definen cada vez más las condiciones de pertenencia al campo del activismo jurídico en derechos humanos. Vale la pena señalar la común referencia a la necesidad de promover y consolidar el Estado de Derecho que ambos segmentos de abogados enarbolan al legitimar sus posiciones en el campo del derecho y de la política.

El artículo de Nicolas Guilhot interroga a aquellos que trabajamos en el contexto local sobre el movimiento por los derechos humanos en un punto nodal respecto de la retórica propia de este activismo y de los valores que lo fundan: el hecho de no instituirse por fuera de la política sino el ser en sí mismo un hecho político. Esto implica, como señala el propio Guilhot:

"cuestionar la narrativa convencional acerca del ascenso de los derechos humanos en un mundo globalizado basado en el activismo transnacional. Lejos de constituir un criterio incuestionable por medio del cual es posible juzgar la política, los derechos humanos son, ellos mismos, un artefacto político. Quisiera sugerir, en particular, que el éxito que hoy tienen los derechos humanos es el resultado de un largo proceso de lucha en términos de apropiaciones, distorsiones, traducciones y de virajes semánticos del concepto que han resultado en una transformación de su sentido y de su campo de aplicación" (énfasis mío).

Las implicancias de esta postura son contundentes: la categoría derechos humanos no preexiste a las luchas por imponer una definición legítima de la misma ya que, en términos de Guilhot:

"Lejos de estar fijado de antemano, el sentido y el contenido de los derechos humanos esboza un espacio semántico trabajosamente elaborado desde su interior por las luchas políticas que tienen por objetivo adquirir la capacidad de imponer una definición legítima de los derechos humanos y, por ende, de obtener los beneficios simbólicos derivados de la posibilidad de subsumir plausiblemente las políticas de Estado bajo esta etiqueta".

Esta línea de trabajo sugiere la importancia de comprender los procesos que han posibilitado la conversión de la vocación por la defensa de los derechos huma- 
nos en una carrera profesional valorada y legitimada en función de capacidades técnicas y no políticas, como sí era el caso a inicios de los años 70, y las implicancias de que sus participantes constituyan una nueva elite jurídica que juega sobre el doble registro de las elites esclarecidas que se atribuyen el lugar de ser la "conciencia moral" de la profesión y de expertos valorados por sus competencias técnicas ocupando posiciones destacadas dentro de la esfera del Estado. Se trata de profesionales del derecho cuya competencia técnica les permite ingresar a espacios privilegiados de actuación profesional local y transnacional en base a su condición de "expertos".

¿Qué implicancias tiene para el investigador asumir esta perspectiva que consiste -como se señalaba al inicio de esta introducción- en trascender las razones tan razonables que explican las acciones de los activistas en derechos humanos y obstinarse en describir a este universo de activismo 'tal como éste es practicado'? O, como señala con acierto Liora Israël en relación con la resistencia a la ocupación alemana, trascender las razones fundadas en el coraje individual, en el valor, el compromiso, la ética y la moral para incluir otras dimensiones de análisis propias de las ciencias sociales? ¿Cómo producir un conocimiento distanciado de las historias sagradas locales que funcionan ellas mismas como referencias claves en la construcción de la propia identidad del investigador y de su grupo de pertenencia? El encantamiento del investigador con el discurso nativo de los familiares y activistas conduce inevitablemente a reproducir la ilusión de un universo militante que se define por oposición al Estado y en función de valores puramente altruistas y desinteresados (Badaró y Vecchioli, 2008).

La desacralización que opera Nicolas Guilhot sobre la temática 'derechos humanos' puede acarrear en el contexto local el riesgo de una acusación moral. Ya que si bien como académicos debidamente entrenados en el relativismo cultural, soportamos la diversidad de puntos de vista siempre y cuando se trate de pueblos lejanos y exóticos, como nos advierte Bourdieu, nos volvemos menos tolerantes cuando los resultados de la investigación sobre nuestra propia sociedad no confirman los supuestos que teníamos sobre temas consustanciales a la definición de nosotros mismos (Bourdieu, 2002: 216). La perspectiva introducida por Guilhot posibilita una redefinición completa de la mirada sobre este universo de relaciones, al poder asociar al activismo en derechos humanos con los procesos de configuración de nuevas elites y espacios de poder.

Apostando a la construcción de una perspectiva comprensiva sobre las relaciones entre derecho y política y con la intención de acompañar los esfuerzos por deslegitimar las pretensiones esencialistas y sociocéntricas sobre el derecho, me interesa concluir esta presentación alentando a los colegas al debate y a la producción de trabajos sobre este campo de actuación a partir de las propuestas sugeridas en esta compilación. 
Profesionales del derecho, activismo jurídico y creación de nuevos derechos...

\section{Bibliografía}

BANCAUD E Yves DeZALAY. 1984. "La sociologie juridique comme enjeu social et professionnel”. Revue Interdisciplinaire d'Études Juridiques 12 : 1-29.

BADARÓ, MÁXimo y Virginia Vecchioli. 2008. "Algunos dilemas y desafíos de una antropología de las elites". En: Etnografías Contemporáneas 4 (4): 7-20.

Bourdieu, Pierre. 1998 (1989). "A força do direito. Elementos para uma sociologia do campo jurídico”. En P. Bourdieu, O poder simbólico. Brasil: Ed. Bertrand Brasil, 209254.

DeZAlay, Yves y BRYANT GARTH. 2008 (1998) "Derechos Humanos y Filantropía Hegemónica". Etnografías Contemporáneas 4 (4): 47-81.

DEZALAY, YVES y BRYANT GARTH. 2002. The internationalization of Palace Wars. Lawyers, Economists and the Contest to Transform Latin American States. Chicago: The University of Chicago Press.

GAÏTI, BRIGITTE y LIORA ISRAËL. 2003. "Sur l'engagement du droit dans la construction des causes". Politix 16 (62) : 17- 30.

Guilhot, NicOlas. 2001. "Les professionnels de la démocratie. Logiques militantes et logiques savantes dans le nouvel internationalisme américain". Actes de la Recherche en Sciences Sociales 139 : 53-65.

LENOIR, REMI. 2002. La fabrique du droit. Une ethnographie du Conseil d' Etat. Paris: La Découverte.

MADSEN, MiKAEL. 2004. "Make law, not war" Les "sociétés impériales" confrontées a l'institutionnalisation internationale des droits de l'homme». Actes de la Recherche en Sciences Sociales 151-152:96-106.

Scheingold, Stuart y Austin Sarat. 2004. Something to Belive. In: Politics, Professionalism and Cause Lawyering. California: Stanford University Press.

VeCCHIOLI, VIRGINIA. 2007. "Derechos Humanos y compromiso militante. Un recorrido por la constitución de esta causa a través de los profesionales del derecho". En: Etnografias Contemporáneas 2 (3): 143- 168. 